\title{
ABBREVIATIONS, ACRONYMS, AND SIGLA
}

AA

$A$ and $E B$

AAS

$A B A$

ABAA

$A B C$

$\mathrm{ABC}$

ABCMA

ABELL

$A B H B$

$A B M I$

$A B P R$

Abrams

ACLA

ACLALSB

$A D B$

$A D D$

ADE

ADFL

$A E B$

AES

AETA

Africa

Africal

AfricaM

AfrL

AfrLS
American Archivist $\mathrm{H}-8$

Analytical and Enumerative

Bibliography Y-38

Asian and African Studies L-148

American Biographical Archive G-39

Antiquarian Booksellers Association of America Y-77

American Book Prices Current $\mathrm{Y}-72$

Association for Business

Communication Z-25

Annotated Bibliography of Canada's Major Authors M-135

Annual Bibliography of English Language and Literature $\mathrm{M}-21$

Annual Bibliography of the History of the Printed Book and Libraries Y-7

Author Biographies Master Index L-31

American Book Publishing Record C-27

see $\mathrm{L}-13$

American Comparative Literature Association Z-25

ACLALSB Bulletin $\mathrm{M}-108$

Australian Dictionary of Biography M-129

American Doctoral Dissertations E-6

Association of Departments of English Z-25

Association of Departments of Foreign Languages Z-10

Analytical and Enumerative Bibliography Y-14, Y-38

AES Abstracts of English Studies $\quad \mathrm{M}-23$

American Educational Theatre Association U-8

Africa: Rivista trimestrale di studie documentzione dell' Istituto italoafricano $\mathrm{L}-138$

Africa: Journal of the International African Institute (London) L-138

Africa (Madrid) L-138

Africana Library Journal L-138

African Language Studies L-138

\begin{tabular}{|c|c|}
\hline AfrS & African Studies L-138 \\
\hline AfrSR & African Studies Review L-138 \\
\hline Afr $-T$ & Africa-Tervuren $\mathrm{L}-138$ \\
\hline$A F S$ & Abstracts of Folklore Studies K-12 \\
\hline$A G R$ & American German Review L-108 \\
\hline AHA & American Historical Association $\mathrm{F}-1$ \\
\hline AHA Guide & AHA Guide to Historical Literature F-1 \\
\hline$A H C$ & Arts and Humanities Citation Index D-19 \\
\hline$A H$ & American Humanities Index D-18 \\
\hline$A H L$ & America, History and Life F-54 \\
\hline$A H R$ & American Historical Review F-10 \\
\hline Ahumor & American Humor S-18 \\
\hline$A I$ & American lmage $\mathrm{X}-38$ \\
\hline$A I C R J$ & $\begin{array}{l}\text { American Indian Culture and Research } \\
\text { Journal S-18 }\end{array}$ \\
\hline$A I Q$ & American Indian Quarterly S-18 \\
\hline AJAS & $\begin{array}{l}\text { Australian Journal of American } \\
\text { Studies S-18 }\end{array}$ \\
\hline AJFS & $\begin{array}{l}\text { Australian Journal of French } \\
\text { Studies L-78 }\end{array}$ \\
\hline$A J P$ & American Journal of Philology $\mathrm{L}-48$ \\
\hline$A L$ & American Literature S-18 \\
\hline ALA & American Library Association \\
\hline$A L H$ & American Literary History S-18 \\
\hline Aling & Analecta Linguistica I-15 \\
\hline Allibone & see $\mathbf{M}-50$ \\
\hline$A L M$ & American Literary Manuscripts $\mathbf{H}-\mathbf{5 1}$ \\
\hline$A L M A$ & Archivum Latinitatis Medii Aevi N-18 \\
\hline$A L R$ & American Literary Realism S-18 \\
\hline$A L S$ & American Literary Scholarship S-14 \\
\hline$A L S$ & $\begin{array}{l}\text { Australian Literary Studies } \mathrm{M}-127 \text {, } \\
\text { M-128 }\end{array}$ \\
\hline ALT & African Literature Today $\mathrm{L}-138, \mathrm{M}-118$ \\
\hline Altick and Wright & see $A-10$ \\
\hline American Catalogue & see $C-28$ \\
\hline AmerS & American Studies S-18 \\
\hline$A m L S$ & American Literary Scholarship S-18 \\
\hline AmSt & Amerikastudien S-18 \\
\hline$A N \& Q$ & American Notes and Queries S-18, Z-58 \\
\hline AnBol & Analecta Bollandiana $\mathrm{N}-18$ \\
\hline
\end{tabular}


AnL

AnM

APHA

APJ

APR

APS

$A Q$

ARBA

Arber

Arcadia

Archiv

Archives

ArielE

ArL

ArmD

AS

ASE

ASECS

ASLIB

ASTR

ATAC

ATESL

ATQ

ATTW

AWN

AWR

$A W W$

Ayers Directory

$B A$

Babel

$B A L$

Baldensperger \&

Friedrich

BALF

BALF

BALLC

BAMBAM

BAR

Baugh

$\boldsymbol{B} \boldsymbol{B}$

$B B$

$B B A$

$B B B$

$B B H$

BBIP

$B B L B$

$B B T$
Anthropological Linguistics 1-15

Annuale Mediaevale $\mathrm{N}-18$

American Printing History

Association Z-25

Academic Publishing Journal Z-107

American Poetry Review T-8

Acta Philologica Scandinavica L-108

American Quarterly S-13, S-18

American Reference Books Annual A-25

see $\mathrm{C}-6, \mathrm{C}-8$

see $L-9$

see $\mathrm{L}-57$

see $\mathrm{H}-8$

Ariel: A Review of International English Literature M-25, M-108

Archivum Linguisticum I-15

Armchair Detective W-8

American Speech I-15, S-18

Anglo-Saxon England $\mathrm{N}-18$

American Society for Eighteenth Century Studies Z-25

Association of Libraries and Information Bureaux B-4

American Society for Theatre Research Z-25

Association of Teachers of Advanced Composition Z-25

Association of Teachers of English as a Second Language Z-25

American Transcendental Quarterly S-18

Association of Teachers of Technical Writing Z-25

Wake Newslitter M-60

Anglo-Welsh Review M-98

American Women Writers L-206

see D-103

Bibliographia Anastatica E-63

see I-106

Bibliography of American Literature S-25

see $\mathbf{L}-1$

Bulletin analytique de linguistique francaise L-74

Black American Literature Forum S-18

Bulletin of the Association for Literary and Linguistic Computing Z-75

Bookline Alert Missing Books and Manuscripts Y-72

Book-Auction Records Y-71

see $\mathbf{M}-30$

Bibliographische Berichte A-3

Bulletin of Bibliography A-7, Y-38

British Biographical Archive G-12

Bibliographie der Buch- und Bibliotheksgeschichte Y-11

Bibliography of British History F-25, F-30, F-32, F-38, F-40, F-43

British Books in Print C-17

Bibliography of British Literary Bibliographies $\mathrm{M}-1$

Bulletin of Black Theater U-18
BBTC

$B C$

$B D M$

$B D P$

Bénet

$B E P I$

Besterman

$B G D S L$

$B H$

BH

BHR

$B H S$

BI

BibFr

Biblio

BIHR

BIHR

BiN

BIP

$B I S$

$B J A$

BJRL

BL

$B L C$

$B L L$

$B L S$

BM

BN

$B N B$

BNIt

BNYPL

BO

Boase

Bompiani

Bonser

Bosworth \& Toller

$B P C$

$B P I$

$B R H$

Brown and Christie

$B R P$

Brunet

BSIM

$B S L P$

BSOAS

BST
British Bibliography and Textual Criticism Y-1

Book Collector Y-38

Biographical Dictionaries Master Index G-50

Beiträge zur deutschen Philologie L-108

see $\mathrm{L}-23$

Bibliography of English Publications in India $\mathrm{M}-154$

$$
\text { see A-1 }
$$

Beiträge zur Geschichte der deutschen Sprache und Literatur L-108

Bulletin hispanique L-95

British Humanities Index D-17

Bibliotheque d' humanisme et Renaissance $\mathrm{N}-18,0-18$

Bulletin of Hispanic Studies L-95

Biography Index G-6

Bibliographie de la France C-54

see C-56

Bulletin of the Institute of Historical Research F-10

Bibliographie internationale de l'humanisme et de la Renaissance O-3

Bibliography Newsletter Y-12, Y-38

Books in Print C-29

Browning Institute Studies M-60

British Journal of Aesthetics X-13

Bulletin of the John Rylands University Library Manchester B-47

British Library B-40

British Library Catalogue of Printed Books to 1975 B-41, D-35

Bulletin linguistscher Literatur I-9

Bulletin of Literary Semiotics X-13

British Museum B-40

Bibliotheque nationale $\mathrm{B}-61$

British National Bibliography C-16

see $\mathrm{C}-73$

Bulletin of the New York Public Library B-30

Black Orpheus: Journal of African and Afro-American Literature M-118

see $\mathrm{G}-11$

see $\mathrm{L}-25$

see F-26

see I-36

Book-Prices Current Y-70

Bookman's Price Index Y-74

Bulletin of Research in the Humanities Z-158

see F-40

Beiträge zur romanischen Philologie L-60, L-63

see C-50

Bibliographie zur Symbolik, Ikonographie und Mythologie K-71

Bulletin de la Sociéte de linguistique de Paris I-15

Bulletin of the School of Oriental and African Studies L-138, L-148

Brontë Society Transactions M-60 


\begin{tabular}{|c|c|}
\hline$B U C O P$ & $\begin{array}{l}\text { British Union Catalogue of } \\
\text { Periodicals D-95 }\end{array}$ \\
\hline$B U L-L$ & $\begin{array}{l}\text { Bibliographie unselbständiger Literatur- } \\
\text { Linguistik I-9 }\end{array}$ \\
\hline BUR & Bucknell Review X-38 \\
\hline Burke & see $G-18, G-19, G-20$ \\
\hline BUSL & $\begin{array}{l}\text { Bibliography of United States } \\
\text { Literature S-11 }\end{array}$ \\
\hline $\mathbf{C}$ & Cambridge University Library B-50 \\
\hline ca. & circa \\
\hline$C A$ & Contemporary Authors R-23 \\
\hline$C A A$ & Contemporary American Authors S-28 \\
\hline$C A L L$ & $\begin{array}{l}\text { Current Awareness Library } \\
\text { Literature } \mathrm{Y}-93\end{array}$ \\
\hline$C A L M$ & $\begin{array}{l}\text { Calendars of American literary } \\
\text { Manuscripts } \mathrm{H}-53\end{array}$ \\
\hline Camb & U.L. Cambridge University Library B-50 \\
\hline CanL & Canadian Literature $\mathrm{M}-143, \mathrm{M}-148$ \\
\hline$C \& L$ & Christianity and Literature $\mathrm{X}-38$ \\
\hline CANR & $\begin{array}{l}\text { Contemporary Authors: New } \\
\text { Revision R-23 }\end{array}$ \\
\hline $\mathrm{CaOTU}$ & University of Toronto Libraries B-39 \\
\hline$C A P$ & $\begin{array}{l}\text { Contemporary Authors: Permanent } \\
\text { Series R-23 }\end{array}$ \\
\hline CAR & $\begin{array}{l}\text { Contemporary Authors: First } \\
\text { Revision R-23 }\end{array}$ \\
\hline Case & see $0-21$ \\
\hline$C B$ & Current Biography G-55 \\
\hline$C B E L$ & $\begin{array}{l}\text { Cambridge Bibliography of English } \\
\text { Literature } \mathrm{M}-10, \mathrm{~N}-10, \mathrm{O}-10, \mathrm{P}-10, \\
\mathrm{Q}-10\end{array}$ \\
\hline CBI & Cumulative Book Index C-30 \\
\hline$C B R I$ & Children's Book Review Index L-179 \\
\hline CCBEL & $\begin{array}{l}\text { Concise Cambridge Bibliography of Eng- } \\
\quad \text { lish Literature } 600-1950 \mathrm{M}-10\end{array}$ \\
\hline$C C C$ & $\begin{array}{l}\text { College Composition and } \\
\text { Communication X-165 }\end{array}$ \\
\hline $\mathrm{CCCC}$ & $\begin{array}{l}\text { Conference on College Composition and } \\
\text { Communication Z-25 }\end{array}$ \\
\hline CCHEL & $\begin{array}{l}\text { Concise Cambridge History of English } \\
\quad \text { Literature } \mathrm{M}-33\end{array}$ \\
\hline$C C L$ & Current Contents Linguistic I-9 \\
\hline$C C L$ & Canadian Children's Literature $\mathrm{L}-179$ \\
\hline$C C M$ & Cahiers de civilisation médiévale $\mathrm{N}-18$ \\
\hline$C C P$ & Catalogue collectif des périoüiques D-98 \\
\hline CCrit & Comparative Criticism: A Yearbook L-9 \\
\hline$C D A B$ & $\begin{array}{l}\text { Concise Dictionary of American } \\
\text { Biography G-35 }\end{array}$ \\
\hline$C D$ & Comprehensive Dissertation Index $\mathrm{E}-5$ \\
\hline$C E$ & College English X-165, Z-25 \\
\hline CEAA & $\begin{array}{l}\text { Center for Editions of American } \\
\text { Authors S-50, Y-36 }\end{array}$ \\
\hline$C E F$ & Catalogue de l' édition française $\quad$ C-57 \\
\hline CEd & Communication Education $\mathrm{X}-165$ \\
\hline CES & $\begin{array}{l}\text { Commonwealth: Essays and } \\
\text { Studies } \mathrm{M}-108\end{array}$ \\
\hline$C H A L$ & $\begin{array}{l}\text { Cambridge History of American } \\
\text { Literature S }-34\end{array}$ \\
\hline ChauR & Chaucer Review N-18 \\
\hline CHBE & $\begin{array}{l}\text { Cambridge History of the British } \\
\text { Empire F-20 }\end{array}$ \\
\hline
\end{tabular}

CHEL

ChildL

ChinL

ChinaQ

CHR

CHUM

CIJE

CJ

CJIS

$C J L$

$C L$

CLA

CLA

CLAJ

Clio

CLS

CLU

CLU-C

CMH

CN

CNRS

Cokayne

COM

ComM

CompD

ComQ

Conch

ConL

ConP

Cooper

CP

$C P$

$C P M$

$C Q$

$C R$

Crane and Kaye

CrevB

CRIS/History

Crit

$C R L$

CRLN

Crum

CS

CSE

CSHVB

$\operatorname{CSmH}$

CTR
Cambridge History of English Literature M-32

Children's Literature: An International Journal L-179

Chinese Literature L-148

China Quarterly L-148

Canadian Historical Review F-10

Computers and the Humanities Z-75

Current Index to Journals in Education Z-125

Classical Journal L-48

Canadian Journal of Irish Studies M-93

Canadian Journal of Linguistics I-15

Comparative Literature $\mathrm{L}-9$

Children's Literature Association Z-25

College Language Association $\mathrm{M}-25$, S-18, Z-25

CLA Journal M-25, S-18

see F-10, X-38

Comparative Literature Studies $\mathrm{L}-9$

University of California, Los Angeles (UCLA) Libraries B-39

William Andrews Clark Memorial Library, UCLA B-39

Cambridge Modern History F-14

Cultura neolatína L-63

Centre national de recherche scientifique I-10

see G-21, G-22

Computer Output Microfiche, Computer Output Microform B-13

Communication Monographs X-165

Comparative Drama U-18

Communication Quarterly X-165

The Conch: A Sociological Journal of African Cultures and Literatures $\mathrm{L}-138$

Contemporary Literature R-18

Contemporary Poetry T-8

see $\mathrm{C}-25$

Classical Philology L-48

Concerning Poetry T-8

Catalogue of Printed Music in the British Library to 1980 B-41

Classical Quarterly L-48

Classical Review L -48

see D-50

Conch Review of Books: A Literary Supplement on Africa L-138

see F-8

Critique: Studies in Modern Fiction R-18, W-8

College and Research Libraries $\quad$ H-8

Comparative Romance Linguistics Newsletter L-63

see $\mathrm{T}-10$

Stanford University Libraries B-39

Center for Scholarly Editions Y-36

Computer Studies in the Humanities and Verbal Behavior Z-75

Henry E. Huntington Library and Art Gallery B-20

Canadian Theatre Review U-18 
CtY

CU

CUBI

D

$D A B$

$D A E$

DAEM

$D A H$

DAI

Davies

$D C B$

Debrett

DeRicci

DFo

DHLR

Dietrich

DLB

DLB

DLC

$D M$

$D N B$

$D O E$

$D Q R$

Drams

DreiN

DSA

Dt

DT

duCangesee

Duff

Dutcher

DVLG

DWL

E\&S

$E A$

$E A$

EAL

EC

$E C C B$

ECF

ECHR

ECLife

$\mathrm{ECr}$

ECS

ECT\&I

ed.

$E E$
Yale University Library B-21, H-27

University of California, Berkeley,

$$
\text { Libraries B-39 }
$$

see C-72

Trinity College, Dublin B-56

Dictionary of American Biography G-35

Dictionary of American English on Historical Principles I-45

Deutsches Archiv für Efforschung des Mittelalters N-18

Dictionary of American History F-57

Dissertation Abstracts International E-7

see F-32

Dictionary of Canadian Biography G-35

$\sec$ G-23

see H-28

Folger Shakespeare Library B-22

D.H. Lawrence Review M-60

see D-13

Dictionary of Literary Biography [Everyman's] M-S1

Dictionary of Literary Biography [Gale] M-58

Library of Congress B-23

Dublin Magazine M-93

Dictionary of National Biography G-10

Dictionary of Old English I-36

Dutch Quarterly Review of AngloAmerican Letters M-25

Drama Survey U-18

Dreiser Newsletter S-50

Dickens Studies Annual: Essays on Victorian Fiction $\mathrm{M}-60, \mathrm{Q}-18$

Trinity College, Dublin B-56

Trinity College, Dublin B-56

I-69

see $\mathrm{C}-4$

see $F-2$

Deutsche Vierteljahresschrift für Literaturwissenschaft und

Geistesgeschichte L-57

Dictionary of World Literature L-11

Essays and Studies M-25

Etudes anglaises M-25

Encyclopedia of Associations Z-28

Early American Literature S-18

Etudes celtiques M-93

Eighteenth Century: A Current Bibliography P-5

Eighteenth-CenturyFiction P-18

Economic History Review F-10

Eighteenth Century Life P-18

L'ésprit créateur L-78

Eighteenth-Century STudies P-18

Eighteenth Century: Theory and

Interpretation P-18

edition

English Education Z-138
EEMSF

EETS

EF

EFT

EG

$E G L$

EHR

E

EIC

EIE

EJ

ELH

ELN

$E L R$

$E L T$

ELT

EngC

enl.

EnlE

ERIC

ES

ESA

ESP

Espasa

ESQ

ESTC

ETC

ETJ

Evans

Expl.

FC

FC

FDP

FEN

FF

FFC

FG

FHA

FID

Filby

FL

$F M$

FMLS

FoLi

Follett

Folklore

Fortescue

Foster

Fowler
Early English Manuscripts in Facsimile $\mathrm{N}-29$

Early English Text Society

Etudes françaises L-78

English Fiction in Transition R-2

Etudes germaniques: Revue trimestrielle L-108

Essay and General Literature Index E-81

English Historical Review F-10

Etudes irlandaises M-93

Essays in Criticism X-13

English Institute X-13

English Journal X-165, Z-138

English Literary History M-25, Q-25

English Language Notes M-25

English Literary Renaissance 0-8, 0-18

English Literature in Transition R-2, R-18

English Language Teaching Journal Z-138

The English Catalogue C-13

enlarged

Enlightenment Essays P-18

Educational Resources Information Center Z-125

English Studies M-25

English studies in Africa M-118

English for Specific Purposes I-15, $\mathrm{X}-165$

see $A-47$

ESQ: A Journal of the American Renaissance S-18,S-50

Eighteenth-Century Short Title Catalogue C-12

Review of General Semantics I-15

Educational Theater Journal U-18

see C-22

Explicator T-8, T-31

Film Comment U-129

Film Criticism U-129

Four Decades of Poetry, 1890-1930 T-8

Freshman English News X-165

Folklore Forum K-23

Folklore Fellows, $F F$

Communications $\mathrm{K}-23$

International Film Guide U-129

Fitzgerald/Hemingway Annual S-50

Fédération international de documentation

see G-14

Foundations of Language I-15

Le français moderne: Revue de linguistique française $\mathrm{L}-78$

Forum for Modern Language Studies L-57

Folia Linguistica I-15

see 1-52

see $\mathrm{K}-23$

see $A-32$

see $\mathbf{G}-28$

see 1-34 


Foxon
$F Q$
$F R$
$F S$
$F S$
FU
G\&R
GAZ
GAZS
GDZS
GEFR
Genre
Georgi
Germanistik
GL
GL
GL\&L
Glossa
Gn
Goff
GQ
GR
Granger's Index
Grässe
Graves
Greg
GRM
Grose
GSLI
GW
GZS

HAHR

Halkett and Laing

\section{Hanham}

Harvey

$H D B$

Heinsius

Hermes

Hinrichs

HIS

Hispania

HJ

\section{HJAS}

HLAS

$H L B$

$H L B$

$H L Q$ see $\mathbf{P}-20$

Film Quarterly U-129

French Review L-78

Feminist Studies L-189

French Studies: A Quarterly Review L-78

University of Florida Libraries B-39

\section{Greece and Rome $\mathrm{L}-48$ \\ Gesamtverzeichnis ausiändischer$$
\text { Zeitschrifien D-98 }
$$

Gesamtverzeichnis ausländischer Zeitschriften und Serien D-98

Gesamtverzeichnis der deutschen Zeitschriften und Serien D-98

George Eliot Fellowship Review M-60

see $\mathrm{L}-9, \mathrm{X}-13$

see $\mathrm{C}-60$

see $\mathbf{L}-108$

General Linguistics I-15

Glotta: Zeitschrift für griechische und lateinische Sprache L-48

German Life and Letters: A Quarterly Review $\mathrm{L}-108$

see I-15

Gnomon: Kritische Zeitschrift für die gesamte klassische Altertumswissenschaft $\mathrm{L}-48$

see $\mathrm{C}-4$

German Quarterly L-108

Germanic Review L-108

see $\mathrm{T}-20$

see $\mathrm{C}-51$

see F-25

see $0-31$

Germanisch-Romanische Monatsschrift L-57

see F-35

Giornale storico della letteratura italiana $\mathbf{L}-\mathbf{8 8}$

Gesamtverzeichnis C-67

Gesamtkatalog der Wiegendrücke C-4

Gesamtverzeichnis der Zeitschrifien und Serien D-98

Hispanic American Historical

$$
\text { Review F-10 }
$$

see E-92

see $\mathrm{F}-43$

see $\mathrm{M}-40$

Hasting's Dictionary of the Bible $\mathrm{K}-41$

see $C-61$

see $\mathrm{L}-48$

see $\mathrm{C}-63$

Humanities in the South Z-158

see L-95

Historical Journal F-10

Harvard Journal of Asiatic Studies L-148

Handbook of Latin American Studies L-99

Harvard Library Bulletin B-26

Huntington Library Bulletin B-20

Huntington Library Quarterly B-20, Z-58
HMC

HMSO

Hoefer

Holman

Howard-Hill

$H Q$

$H R$

HRHRC

HSCP

HudR

Hyamson

IBBB

IBF

IBHS

IBN

IBR

IBR

IBRH

IBT

IBZ

ICN

ICU

$I D B$

IEN

IF

IFR

IHS

IJAL

IJBF

IJOH

IJPS

IJSL

IJSLP

IJSym

IMB

IMEP .

IMEV

IMU

IncL

IndL

IndLing

InU
Historical Manuscripts Commission $H-60, H-61$

Her Majesty's Stationery Office

see G-9

see $\mathrm{L}-12$

see $\mathrm{M}-1,0-45, \mathrm{Y}-1$

Hopkins Quarterly M-60

Hispanic Review L-95

Harry Ransom Humanities Research Center B-35

Harvard Studies in Classical Philology L-48

Hudson Review Z-158

see $\mathrm{G}-2$

Internationale Bibliographie des Buch-und Bibliothekswesens Y-8

Internationale Bibliographie der Festschriften E-85

International Bibliography of Historical Sciences F-4

Index Bio-bibliographicus Notorum Hominum G-5

Internationale Bibliographie der Reprints E-62

Internationale Bibliographie der Rezensionen E-75

Index to Book Reviews in the Humanities E-73

International Bibliography of Theatre U-15

Internationale Bibliographie der Zeitschriftenliteratur D-13

Newberry Library B-25

University of Chicago Libraries B-39

Interpreter's Dictionary of the Bible $\mathrm{K}-40$

Northwestern University Libraries B-39

Indogermanische Forschungen $\mathrm{L}-108$

International Fiction Review W-8

Irish Historical Studies M-93

International Journal of American Linguistics 1-15

Internationale Jahres-Bibliographie der Festschriften E-85

International Journal of Oral History F-10

International Journal of Psycholinguistics I-15

International Journal of the Sociology of Language I-15

International Journal of Slavic Linguistics and Poetics $\mathrm{L}-128$

International Journal of Symbology K-79

International Medieval Bibliography F-23

Index of Middle English Prose $\mathrm{N}-38$

Index of Middle English Verse N-36, $\mathbf{N}-\mathbf{3 7}$

Italia medioevale e umanistica $\quad \mathrm{L}-\mathbf{8 8}$

Incorporated Linguist I-15, I-106

Indian Literature $\mathrm{L}-148, \mathrm{M}-158$

India Linguistics $\mathrm{M}-158$

University of Indiana Libraries B-39 


\begin{tabular}{|c|c|c|c|}
\hline IRAL & $\begin{array}{l}\text { International Review of Applied } \\
\text { Linguistics I-15 } \\
\text { Italnianistica: rivista di letteratura }\end{array}$ & $\begin{array}{l}\text { JPSyR } \\
\text { JRAS }\end{array}$ & $\begin{array}{c}\text { Journal of Psycholinguistic Research I-15 } \\
\text { Journal of the Royal Asiatic Society of } \\
\text { Great Britain and Ireland L-148 }\end{array}$ \\
\hline & italiana $\mathbf{L}-88$ & $J R S$ & Journal of Roman Studies L-48 \\
\hline IS & Italian Studies L-88 & JSOAL & Journal of South Asian Literature L-148, \\
\hline $\begin{array}{l}\text { ISA } \\
\text { ISRN }\end{array}$ & Irregular Series and Annuals D-101 & $J S S E$ & $\begin{array}{l}\text { M-158 } \\
\text { Journal of the Short Story in English W-8 }\end{array}$ \\
\hline $\begin{array}{l}\text { ISBN } \\
\text { ISSHP }\end{array}$ & $\begin{array}{l}\text { International Standard Book Number } \\
\text { Index to Social Science and Humanities } \\
\text { Proceedings E-88 }\end{array}$ & $\begin{array}{l}\text { SSEE } \\
J T W C\end{array}$ & $\begin{array}{l}\text { Journal of Technical Writing and } \\
\text { Communication X-165 }\end{array}$ \\
\hline $\begin{array}{l}\text { Italica } \\
\text { IU }\end{array}$ & $\begin{array}{l}\text { see L-88 } \\
\text { University of Illinois Libraries B-39 }\end{array}$ & JUFVA & $\begin{array}{l}\text { Journal of the University Film and Video } \\
\text { Association U-129 }\end{array}$ \\
\hline IUR & Irish University Review M-93 & $J W C I$ & $\begin{array}{l}\text { Journal of the Warburg and Courtauld } \\
\text { Instituses } \mathrm{X}-\mathbf{3 8}\end{array}$ \\
\hline$J A A C$ & $\begin{array}{l}\text { Journal of Aesthetics and Art } \\
\text { Criticism X-13, X-32, X-38 }\end{array}$ & $J W S L$ & $\begin{array}{l}\text { Journal of Women's Studies in } \\
\text { Literature L-188 }\end{array}$ \\
\hline$J A C$ & Journal of Advanced Composition X-165 & & \\
\hline$J A C$ & Journal of American Culture S-18 & Kayser & $\sec C-62$ \\
\hline JAF & Journal of American Folklore $\mathrm{K}-23$ & Kelly & see C-26 \\
\hline $\boldsymbol{J} A \boldsymbol{H}$ & Journal of American History F-10 & Kenyon \& Knott & see I-53 \\
\hline Jahresverzeichnis & see E-18 & Klapp & see $L-73$ \\
\hline $\begin{array}{l}\text { JAmS } \\
\text { JAOS }\end{array}$ & $\begin{array}{l}\text { Journal of American Studies S-18 } \\
\text { Journal of the American Oriental }\end{array}$ & KSGT & $\begin{array}{l}\text { Kleine Schrifien der Gesellschaft fur } \\
\text { Theatergeschichte U-18 }\end{array}$ \\
\hline & Society L-148 & $K S J$ & Keats-Shelley Journal M-60, Q-18, Q-26 \\
\hline JapQ & Japan Quarterly L-148 & $K S M B$ & $\begin{array}{l}\text { Keats-Shelley Memorial Bulletin. } \\
\text { Rome. M-60, Q-18 }\end{array}$ \\
\hline $\begin{array}{l}\text { JAsial } \\
\text { JASt }\end{array}$ & $\begin{array}{l}\text { Journal asiatique L-148 } \\
\text { Journal of Asian Studies L-148 }\end{array}$ & L & British Library B-40 \\
\hline JBRS & $\begin{array}{l}\text { Journal of the Burma Research } \\
\text { Society L-148 }\end{array}$ & $\begin{array}{l}L \& H \\
L \&\end{array}$ & $\begin{array}{l}\text { Literature and History } \quad \mathrm{X}-38 \\
\text { Literature and Ideology } \quad \mathrm{X}-38\end{array}$ \\
\hline$J B S$ & Journal of British Studies F-10 & $L \& P$ & Literature and Psychology $\mathrm{X}-38, \mathrm{X}-61$ \\
\hline$J C$ & Journal of Communication X-165 & $L \& S$ & Language and Speech I-15 \\
\hline $\begin{array}{l}J C F \\
J C L\end{array}$ & $\begin{array}{l}\text { Journal of Canadian Fiction M-148, W-8 } \\
\text { Journal of Commonwealth }\end{array}$ & $L \& U$ & $\begin{array}{l}\text { Lion and the Unicorn: A Critical Journal of } \\
\text { Children's Literature L-179 }\end{array}$ \\
\hline & Literature L-138, M-101, M-108 & $L A$ & Language Arts $\mathrm{Z}-138$ \\
\hline$J C P$ & Journal of Canadian Poetry M-148 & $L A L R$ & Latin American Literary Review L-95 \\
\hline JEGP & $\begin{array}{l}\text { Journal of English and Germanic } \\
\text { Philology L-108, L-118 }\end{array}$ & Lang\&S & $\begin{array}{l}\text { Language and Style: An International } \\
\text { Journal I-15 }\end{array}$ \\
\hline$J E H$ & Journal of Economic History F-10 & Langer & see F-11 \\
\hline$J E n g L$ & Journal of English Linguistics I-15 & Language & see $\mathbf{I}-15$ \\
\hline$J E S$ & Journal of Ethnic Studies S-18 & Latham & see $1-69$ \\
\hline$J F I$ & Journal of the Folklore Institute $\mathrm{K}-23$ & LATR & Latin-American Theater Review U-18 \\
\hline $\begin{array}{l}J G E \\
J H\end{array}$ & $\begin{array}{l}\text { Journal of General Education Z-138 } \\
\text { Journal of the History of Ideas F-10, }\end{array}$ & $\mathbf{L C}$ & $\begin{array}{l}\text { Library of Congress A-33, B-12, B-13, } \\
\text { H-23 }\end{array}$ \\
\hline & $\mathrm{X}-38$ & $L C R$ & Literary Criticism Register M-24 \\
\hline $\begin{array}{l}J H S \\
J I L\end{array}$ & $\begin{array}{l}\text { Journal of Hellenic Studies } \quad \mathrm{L}-\mathbf{4 8} \\
\text { Journal of Irish Literature } \mathrm{M}-\mathbf{9 3}\end{array}$ & $L C S H$ & $\begin{array}{l}\text { Library of Congress Subject } \\
\text { Headings A-33 }\end{array}$ \\
\hline$J \boldsymbol{J} Q$ & James Joyce Quarterly M-60, M-93 & $L E \& W$ & Literature East and West L-148 \\
\hline$J J S$ & Journal of Japanese Studies $\quad$ L-148 & Lewis \& Short & see I-65 \\
\hline$J K S$ & Journal of Korean Studies L-148 & $L F Q$ & Literature/Film Quarterly U-129 \\
\hline$J L$ & Journal of Linguistics I-15 & LHUS & $\begin{array}{l}\text { Literary History of the United } \\
\text { States S-10, S-30 }\end{array}$ \\
\hline $\begin{array}{l}J L S \\
J M H\end{array}$ & $\begin{array}{l}\text { Journal of Literary Semantics } \quad I-15 \\
\text { Journal of Modern History F-10 }\end{array}$ & $L H Y$ & Literary Half-Yearly $\mathrm{M}-158$ \\
\hline$J M L$ & $\begin{array}{l}\text { Journal of Modern History F-10 } \\
\text { Journal of Modern Literature R-17, R-18 }\end{array}$ & $L$ & Lettere italiane $\mathbf{L}-88$ \\
\hline JMRS & $\begin{array}{l}\text { Journal of Medieval and Renaissance } \\
\text { Studies N-18 }\end{array}$ & $\begin{array}{l}\text { Liddell \& Scott } \\
\text { LIMC }\end{array}$ & $\begin{array}{l}\text { see I-60 } \\
\text { Lexicon Iconographicum Mythologiae } \\
\quad \text { Classicae K-29 }\end{array}$ \\
\hline$J N L$ & $\begin{array}{l}\text { Johnsonian Newsletter M-60, P-9, P-18, } \\
\text { Z-58 }\end{array}$ & Ling \& $P$ & Linguistics and Philosophy I-15 \\
\hline$J N T$ & Journal of Narrative Technique $\quad$ W-8 & LingA & Linguistic Analysis I-15 \\
\hline Jones & see I-35 & Lingl & Linguistic Inquiry I-15 \\
\hline$J P C$ & Journal of Popular Culture K-23 & Lingua & see $1-15$ \\
\hline JPS & Journal of the Polynesian Society M-168 & $\begin{array}{l}\text { Linguistics } \\
\mathrm{L}\end{array}$ & $\begin{array}{l}\text { see I-15 } \\
\text { Library Journal Y-98 }\end{array}$ \\
\hline
\end{tabular}




\begin{tabular}{|c|c|c|c|}
\hline $\begin{array}{l}L L \\
L L B A\end{array}$ & $\begin{array}{l}\text { Language Learning I-15 } \\
\text { Language and Language Behavior }\end{array}$ & $M N$ & $\begin{array}{l}\text { Monumensa Nipponica: Studies in Japa- } \\
\text { nese Culture L-148 }\end{array}$ \\
\hline & Abstracts I-7 & $\mathrm{MnU}$ & University of Minnesota Libraries B-39 \\
\hline \multirow[t]{2}{*}{ LUINQUA } & Language and Literature Index & Monatshefie & see $L-108$ \\
\hline & Quarterly L-7 & Mosaic & $\sec L-9$ \\
\hline LNHT & Tulane University Libraries B-39 & Mosw & Washington University Libraries \\
\hline$L N L$ & Linguistics in Literature $\mathrm{X}-165$ & & see $\mathrm{H}-27$ \\
\hline Lodge & $\sec G-24$ & Motif-Index & see $K-2$ \\
\hline LondC & The London Catalogue C-11 & $M P$ & Modern Philology L-57 \\
\hline$\angle O P$ & Language of Poetry $\mathrm{T}-8$ & MPS & Modern Poetry Studies T-8 \\
\hline Lorenz & see $C-55$ & MS & manuscript \\
\hline Lowndes & see $\mathrm{C}-3$ & $M S$ & Mediaeval Studies $\quad \mathrm{N}-18$ \\
\hline$L Q$ & Library Quarterly Y-38 & MSEX & Melville Society Extracts S-50 \\
\hline $\boldsymbol{L R}$ & Les Lettres romanes L-63 & MSS & manuscripts \\
\hline$L R$ & $\begin{array}{l}\text { Literary Research: A Journal of Scholarly } \\
\text { Method and Technique Y-14 }\end{array}$ & $\begin{array}{l}\text { MSS } \\
\text { MTJ }\end{array}$ & $\begin{array}{l}\text { Manuscripts H-8 } \\
\text { Mark Twain Journal S-50 }\end{array}$ \\
\hline $\boldsymbol{L R B}$ & London Review of Books Z-153 & $M T L A$ & Micropublisher's Trade List Annual E-52 \\
\hline LSA & Linguistic Society of America Z-25 & MuK & Maske and Kothurn U-18 \\
\hline \multirow[t]{2}{*}{ LSoc } & Language in Society I-15 & Muret-Sanders & see $1-85$ \\
\hline & & Muséon & see $L-148$ \\
\hline $\boldsymbol{M A}$ & Le moyen age $\mathrm{N}-18$ & MWA & American Antiquarian Society \\
\hline$M A E$ & Medium Aevum N-18 & & Library B-27, H-27 \\
\hline $\begin{array}{l}\text { MAL } \\
\text { Malcless }\end{array}$ & $\begin{array}{l}\text { Modern Austrian Literature } \mathrm{L}-108 \\
\text { see A-22 }\end{array}$ & MWH & $\begin{array}{l}\text { Massachusetts Historical Society } \\
\text { Library H-27 }\end{array}$ \\
\hline$M \& H$ & Medievalia et Humanistica N-18 & & \\
\hline Manuscripta & see $\mathrm{H}-8$ & NAIP & $\begin{array}{l}\text { North American Imprints } \\
\text { Programsee C-12 }\end{array}$ \\
\hline MARC & Machine readable cataloging $\mathrm{Z}-78$ & Names & see $1-15$ \\
\hline MARC & Machine Readable Cataloging see Z-78 & $N \& Q$ & Notes and Queries Z-58 \\
\hline $\begin{array}{l}\text { Mathews } \\
\text { MB }\end{array}$ & $\begin{array}{l}\text { see I-46 } \\
\text { Boston Public Library B-39, H-27 }\end{array}$ & NCBEL & New Cambridge Bibliography of English \\
\hline $\begin{array}{l}\text { MB } \\
\text { McGuire }\end{array}$ & $\begin{array}{l}\text { Boston Public Library B-39, H-27 } \\
\text { see L-40 }\end{array}$ & & $\begin{array}{l}\text { Literature M-11, N-11, O-11, P-11, } \\
Q-11, \mathrm{R}-11\end{array}$ \\
\hline McNamee & see $E-20$ & $\mathrm{NcD}$ & Duke University Libraries B-39 \\
\hline$M D$ & Modern Drama R-18, R-44 & $N C F$ & Nineteenth Century'Fiction $Q-18, W-8$ \\
\hline$M D A C$ & Mystery and Detection Annual W-8 & $N C M H$ & New Cambridge Modern History F-14 \\
\hline MdHi & Maryland Historical Society Library H-27 & NConL & Notes on Contemporary Literature R-18 \\
\hline MED & Middle English Dictionary $\mathbf{1 - 3 8}$ & NCSTC & Nineteenth Century Short Title \\
\hline MeWC & Colby College Library B-39 & & Catalogue $\mathrm{C}-14$ \\
\hline MFS & Modern Fiction Studies R-18, R-59, W-8 & NCTE & National Council of Teachers of \\
\hline MH & Harvard University Libraries B-26, H-27 & & English Z-25, Z-129 \\
\hline MHRA & $\begin{array}{l}\text { Modern Humanities Research } \\
\text { Association L-52, M-21, Z-25, Z-94 }\end{array}$ & NCTR & $\begin{array}{l}\text { Nineteenth Century Theatre } \\
\text { Research } Q-18, Q-62, \mathrm{U}-18\end{array}$ \\
\hline Michaud & see G-9 & $\mathrm{NcU}$ & University of North Canolina B-39 \\
\hline MIO & Mitteilungen des Instituts für & $N D B$ & Neue deutsche Biographie G-61 \\
\hline MissQ & $\begin{array}{r}\text { Orientforschung } \mathrm{L}-148 \\
\text { Mississippi Quarterly S-18 }\end{array}$ & $N E D$ & $\begin{array}{l}\text { New English Dictionary on Historical } \\
\text { Principles I-33 }\end{array}$ \\
\hline MiU & $\begin{array}{l}\text { University of Michigan Libraries B-39, } \\
\text { H-27 }\end{array}$ & NEMLA & $\begin{array}{l}\text { Northeast Modern Language } \\
\text { Association Z-25 }\end{array}$ \\
\hline MiU-C & Clements Library, University of & Neohelicon & see $L-9$ \\
\hline & Michigan B-39, H-27 & Neophil. & Neophilologus L-57 \\
\hline MLA & Modern Language Association L-50, & $N E Q$ & New England Quarterly S-18, S-101 \\
\hline$M L A I B$ & $\begin{array}{l}\text { Z-25, Z-94 } \\
\text { MLA International Bibliography L-50 }\end{array}$ & $N H J$ & Nathaniel Hawthorne Journal S-50 \\
\hline$M L J$ & $\begin{array}{l}\text { MLA International Bibliography L-50 } \\
\text { Modern Language Journal L-57 }\end{array}$ & NHPRC & $\begin{array}{l}\text { National Historical Publications and Rec- } \\
\text { ords Commission H-21 }\end{array}$ \\
\hline$M L N$ & $\begin{array}{l}\text { MLN: Modern Language Notes L-57, } \\
\text { Z-58 }\end{array}$ & $\begin{array}{l}\text { NIC } \\
\text { Nicholson }\end{array}$ & $\begin{array}{l}\text { Cornell University Libraries B-39 } \\
\text { see I-51 }\end{array}$ \\
\hline$M L Q$ & Modern Language Quarterly L-57 & Nilon & see $S-1$ \\
\hline$M L R$ & Madern Language Review L -57 & NIM & Newspapers in Microform E-56 \\
\hline$M L S$ & Modern Language Studies L-57 & NjP & Princeton University Libraries B-39, \\
\hline MMLA & Midwest Modem Language & & H-27 \\
\hline & Association $2-25$ & NLH & New Literary History $\mathrm{X}-13$ \\
\hline & & NLI & National Library of Ireland B-57 \\
\hline
\end{tabular}




\begin{tabular}{|c|c|c|c|}
\hline NLS & National Library of Scotland B-54 & Paetow & see $F-21$ \\
\hline NLW & National Library of Wales B-53 & Pagliani & see $C-71$ \\
\hline$N M$ & $\begin{array}{l}\text { Neuphilologische Mitteilungen L-57, } \\
\text { N-18 }\end{array}$ & $\begin{array}{l}\text { Palmer's Index } \\
\text { Panzer }\end{array}$ & $\begin{array}{ll}\text { see } D-21 \\
\text { see } C-5\end{array}$ \\
\hline $\begin{array}{l}\text { NN } \\
\text { NNC }\end{array}$ & $\begin{array}{l}\text { New York Public Library B-30, H-27 } \\
\text { Columbia University Libraries B-39, }\end{array}$ & PAPC & $\begin{array}{l}\text { Philological Association of the Pacific } \\
\text { Coast } \mathrm{Z}-25\end{array}$ \\
\hline & $\mathrm{H}-27$ & $P A R$ & Performing Arts Resources U-18 \\
\hline NNPM & Pierpont Morgan Library B-31, H-27 & Pargellis and Medley & see $F-38$ \\
\hline NNU & New York University Libraries B-39 & Partridge & see $I-42$ \\
\hline $\begin{array}{l}\text { NPT } \\
\text { NRA }\end{array}$ & $\begin{array}{l}\text { New Periodical Titles D-15 } \\
\text { National Register of Archives H-62 }\end{array}$ & $P B S A$ & $\begin{array}{l}\text { Papers of the Bibliographical Society of } \\
\text { America Y-38 }\end{array}$ \\
\hline NRam & $\begin{array}{l}\text { New Rambler: Journal of the Johnson Soci- } \\
\text { ety of London M-60, P-18 } \\
\text { Nouvelle revue française X-13 }\end{array}$ & $\begin{array}{l}\text { Peddie } \\
P E P P\end{array}$ & $\begin{array}{l}\text { see A-31 } \\
\text { Princeton Encyclopedia of Poetry and } \\
\quad \text { Poetics L-21 }\end{array}$ \\
\hline NRFH & $\begin{array}{l}\text { Nueva revista de filologı́ hispánica L-93, } \\
\text { L-95 }\end{array}$ & $\begin{array}{l}\text { Phaedrus } \\
\text { PLL }\end{array}$ & $\begin{array}{l}\text { see } \mathrm{L}-178 \\
\text { Papers on Language and Literature L-57 }\end{array}$ \\
\hline$N R M M$ & $\begin{array}{l}\text { National Register of Microform } \\
\text { Masters E-53 }\end{array}$ & $P M L A$ & $\begin{array}{l}\text { PMLA: Publications of the Modern Lan- } \\
\text { guage Association of America L-57 }\end{array}$ \\
\hline NS & Die Neueren Sprachen L-57 & PoeS & Poe Studies S-50 \\
\hline NST & New Serial Titles D-92 & Pollard and Redgrave & see $\mathrm{C}-5$ \\
\hline NSTC & New Short Title Catalogue C-5 & Poole's Index & see $D-10$ \\
\hline $\begin{array}{l}\text { NUC } \\
\text { NUCMC }\end{array}$ & $\begin{array}{l}\text { National Union Catalog B-12, B-13 } \\
\text { National Union Catalog of Manuscript }\end{array}$ & $P P$ & $\begin{array}{l}\text { Past and Present: A Journal of Historical } \\
\text { Studies F-10 }\end{array}$ \\
\hline & Collections $\mathbf{H}-22$ & $P P I$ & Popular Periodical Indexsee D-15 \\
\hline $\begin{array}{l}N Y F Q \\
N Y R B\end{array}$ & $\begin{array}{l}\text { New York Folklore Quarterly K-23 } \\
\text { New York Review of Books Z-152 }\end{array}$ & PPL & $\begin{array}{l}\text { The Library Company of } \\
\text { Philadelphia B-39 }\end{array}$ \\
\hline NYTBR & New York Times Book Review Z-150 & $\begin{array}{l}P Q \\
P Q M\end{array}$ & $\begin{array}{l}\text { Philological Quarterly L-57, P-5 } \\
\text { Pacific Quarterly M-168 }\end{array}$ \\
\hline $\mathbf{O}$ & Bodleian Library B-48 & $P R$ & Partisan Review Z-158 \\
\hline$O A D$ & Oxford American Dictionary I-50 & PRECIS & Preserved Context Index System A-32 \\
\hline$O C A L$ & $\begin{array}{l}\text { Oxford Companion to American } \\
\text { Literature S-40 }\end{array}$ & $\begin{array}{l}\text { PRO } \\
P / T\end{array}$ & $\begin{array}{l}\text { Public Records Office H-70, H-71 } \\
\text { Pre/Text X-165 }\end{array}$ \\
\hline OCCL & $\begin{array}{l}\text { Oxford Companion to Classical } \\
\text { Literature L-42 }\end{array}$ & $P T L$ & $\begin{array}{l}\text { PTL: A Journal for Descriptive Poetics and } \\
\text { Theory of Literature } \mathrm{X}-13\end{array}$ \\
\hline$O C D$ & Oxford Classical Dictionary L-42 & $P T L A$ & Publishers Trade List Annual C-29 \\
\hline$O C E L$ & $\begin{array}{l}\text { Oxford Companion to English } \\
\text { Literature } \mathrm{M}-40\end{array}$ & PU & University of Pennsylvania Libraries B-39 \\
\hline$O C F L$ & $\begin{array}{l}\text { Oxford Companion to French } \\
\text { Literature L-71 }\end{array}$ & & 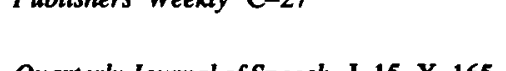 \\
\hline OCGL & $\begin{array}{l}\text { Oxford Companion to German } \\
\text { Literature } \mathrm{L}-111\end{array}$ & $\begin{array}{l}Q J S \\
Q R F S\end{array}$ & $\begin{array}{l}\text { Quarterly Journal of Speech } 1-15, \text { X-165 } \\
\text { Quarterly Review of Film Studies U-129 }\end{array}$ \\
\hline OCLC & Online Computer Library Center Z-78 & Quérard & see $\mathrm{C}-52$ \\
\hline OCSL & $\begin{array}{l}\text { Oxford Companion to Spanish } \\
\text { Literature L-91 } \\
\text { Oxford English Dictionary on Historical }\end{array}$ & $R A L$ & $\begin{array}{l}\text { Research in African Literatures L-138, } \\
\quad \mathrm{M}-118\end{array}$ \\
\hline OENews & $\begin{array}{l}\text { Principles I-33 } \\
\text { Old English Newsletter N-18, N-22 }\end{array}$ & RALF & $\begin{array}{l}\text { Repetoire analytique de littérature } \\
\text { française } \mathrm{L}-74\end{array}$ \\
\hline OHEL & $\begin{array}{l}\text { Oxford History of English } \\
\text { Literature } \mathrm{M}-31\end{array}$ & $R A L S$ & $\begin{array}{l}\text { Resources for American Literary } \\
\text { Study S-15 }\end{array}$ \\
\hline OJES & $\begin{array}{l}\text { Journal of English Studies } \\
\quad \text { (Osmania) } \mathrm{M}-158\end{array}$ & $\begin{array}{l}\text { Rancoeur } \\
R B\end{array}$ & $\begin{array}{l}\text { see } \mathrm{L}-74 \\
\text { Revue bénédictine } \mathrm{N}-18\end{array}$ \\
\hline $\begin{array}{l}O L \\
O L D\end{array}$ & $\begin{array}{l}\text { Orbis Litterarum L-9 } \\
\text { Oxford Latin Dictionary } 1-66\end{array}$ & $R-E$ & $\begin{array}{c}\text { Real-Encyclopädie der Klassischen } \\
\text { Altertumswissenschafi } \mathrm{L}-41\end{array}$ \\
\hline Onoma & see I-15 & $R E A$ & Revue des études anciennes $\quad$ L-48 \\
\hline$\infty$ & Oberlin College Library B-39 & Read & see $F-30$ \\
\hline $\begin{array}{l}\text { Orbis } \\
\text { Orientalia }\end{array}$ & $\begin{array}{ll}\text { see } I-15 \\
\text { see } L-148\end{array}$ & RECTR & $\begin{array}{l}\text { Restoration and Eighteenth Century The- } \\
\text { atre Research } \mathrm{P}-18, \mathrm{P}-31, \mathrm{U}-18\end{array}$ \\
\hline OU & Ohio State University Libraries B-39 & REED & Records of Early English Drama N-54 \\
\hline & Philosophy and & REEDN & $\begin{array}{c}\text { Records of Early English Drama } \\
\text { Newsletter } \mathrm{N}-54, \mathrm{U}-18\end{array}$ \\
\hline$P \& R$ & Philosophy and Rhetoric X-165 & $R E G$ & Revue des études grecques $\quad$ L-48 \\
\hline$P A$ & Présence africaine $\mathrm{L}-138$ & $\begin{array}{l}R E H \\
R E I\end{array}$ & $\begin{array}{l}\text { Revista de estudios hispánicos L-95 } \\
\text { Revue des études italiennes L-88 }\end{array}$ \\
\hline
\end{tabular}


REL

RELO

Ren\&R

RenD

$\operatorname{Ren} Q$

RER

RES

rev.

RF

RFE

RFI

RFR

$R G$

RHL

RHM

Rht

RIB

RIE

RILA

RILM

RJ

$R L$

RL

$R L I$

RLIN

RLiR

$R L J$

RLMC

$R L T$

$R L V$

RMS

RNL

Romania

RomN

Roorbach

RORD

$\boldsymbol{R P}$

$\boldsymbol{R P A}$

RPB

$R P h$

RPP

$R Q$

$R R$

$R \boldsymbol{R}$

$R R Q$

RSQ

RSTC

RSVP
Revue des études latines $\quad \mathrm{L}-48$

Revue de l'Organization internationale pour l' etude des langues anciennes par ordinateur Z-75

Renaissance and Reformation/Renaissance et Réforme O-18

Renaissance Drama 0-18

Renaissance Quarterly 0-6, 0-18

Review of Educational Research Z-138

Review of English Studies M-25

revised

Romanische Forschungen L-63

Revista de filologia española L-95

Regionalism and the Female Imagination $\mathrm{L}-189$

Resources for Feminist Research L-187

Reader's Guide to Periodical Literature

$$
\text { D-15 }
$$

Révue d' histoire littéraire de la France $\mathbf{L}-\mathbf{7 4}, \mathrm{L}-\mathbf{7 8}$

Revista Hispania moderna L-95

Revue d' histoire du theatre U-18

Revista interamericana de bibliografia L-95

Resources in Education Z-126

Répertoire internationale de la littérature de l'art X-45

Répertoire internationale de littérature musicale $\mathrm{X}-40$

Romanistisches Jahrbuch L-63

Revista de literatura L-95

Revue de linérature comparée L-9

Rassegna della letteratura italiana L -88

Research Libraries Information Network Z-78

Revue de linguistique romane L-63

Russian Language Journal L-128

Rivista di litterature moderne e comparate L-9

Russian Literature Tri-quarterly L-128

Revue des langues vivantes L-57

Renaissance and Modern Studies O-18

Review of National Literatures L-9

see $\mathbf{L}-63$

Romance Notes L-63

see C-24

Research Opportunities in Renaissance Drama 0-18, 0-33, U-18

Romanische Bibliographie L-60

Recently Published Articles [in Historyl F-6

Brown University Libraries B-33

Romance Philology L-63

Romanticism Past and Present Q-18

Reference Quarterly Y-38

Rhetoric Review X-165

Romanic Review L-63

Reading Research Quarterly Z-138

Rhetoric Society Quarterly X-106, X-165

Revised Short Title Catalogue C-5

Research Society for Victorian Periodicals Q-18, Q-48, Z-25
RTE

RusL

RusLing

RusR

$S \& S$

$S \& S$

$S A$

$S A B$

$S A B$

Sabin

Sader

$S A F$

$S A I L$

SAMLA

$S A Q$

$S B$

$S B H C$

$S B L$

SBTC

SCA

Scan

ScanR

$S C L$

SCMLA

SCN

SCraN

ScS

SCT

SECC

SEEJ

SEER

$S E L$

Semiotica

SFBRI

SFNL

$S F Q$

SFr

SFS

$S H$

Shaks

Shaw

ShawR

Sheehy

Shipley

ShJ

ShJE

$S h / W$

$S h N$

Shoemaker
Research in the Teaching of

English X-165, Z-129, Z-138

Russian Literature L-128

Russian Linguistics L-128

Russian Review L-128

Science and Society $\mathrm{X}-38$

Sight and Sound U-129

Studi Americani S-18

Shakespeare Association Bulletin 0-58

South Atlantic Bulletin L-57

see C-21

see D-29

Studies in American Fiction S-18, W-8

Studies in American Indian Literature S-18

South Atlantic Modern Language Association Z-25

South Atlantic Quarterly Z-158

Studies in Bibliography Y-38

Studies in Browning and His Circle $\mathrm{M}-60$

Studies in Black Literature M-118, S-18

Shakespearean Bibliography and Textual Criticism O-45

Speech Communication Association Z-25

Scandinavica L-108

Scandinavian Review L-108

Studies in Canadian Literature M-148

South Central Modern Language Association Z-25

Seventeenth Century News 0-9, 0-18, P-18

Stephen Crane Newsletter S-50

Scottish Studies M-78

Schriften der Gesellschafi fur Theatergeschicte U-18

Studies in Eighteenth Century Culture P-18

Slavic and East European Journal L-128

Slavonic and East European Review L-128

Studies in English Literature $\mathrm{M}-25,0-7$, $0-18,0-32, \mathrm{P}-4, \mathrm{P}-18, \mathrm{Q}-5$

see I-15

Science Fiction Book Review Index W-108

Shakespeare on Film Newsletter 0-58

Southern Folklore Quarterly K-23

Studi francesi L-78

Science Fiction Studies W-8

Studia Hibernica M-93

Shakespeare Studies O-58

see $\mathrm{C}-23$

Shaw Review M-60

see A-20

see L-11

Shakespeare-Jahrbuch 0-58

Shakespeare Jahrbuch (East) O-58

Shakespeare Jahrbuch (West) O-58

Shakespeare Newsletter 0-53, 0-58

see $\mathbf{C}-25$ 
XXviii Abbreviations, Acronyms, and Sigla

\begin{tabular}{|c|c|c|c|}
\hline $\begin{array}{l}\text { SHR } \\
\text { ShS }\end{array}$ & $\begin{array}{l}\text { Scottish Historical Review F-10 } \\
\text { Shakespeare Survey O-58 }\end{array}$ & TCBS & $\begin{array}{l}\text { Transactions of the Cambridge Biblio- } \\
\text { graphical Society Y-38 }\end{array}$ \\
\hline ShStud & Shakespeare Studies (Tokyo) 0-58 & $T C L$ & Twentieth Century Literature $\mathrm{R}-16, \mathrm{R}-18$ \\
\hline SIGLASH & $\begin{array}{l}\text { Newsletter of the Special Interest Group on } \\
\text { Language Analysis and Studies in the } \\
\text { Humanities } \mathrm{Z}-75\end{array}$ & $\begin{array}{l}\text { TDR } \\
\text { Term Catalogues } \\
\text { TESOL }\end{array}$ & $\begin{array}{l}\text { The Drama Review R-18, U-18 } \\
\text { see C-8 } \\
\text { Teachers of English to Speakers of Other }\end{array}$ \\
\hline Signs & see L-189 & & Languages Z-25 \\
\hline SIP & Subject Index to Periodicals see D-17 & TETYC & Teaching English in the Two-Year \\
\hline SIR & Studies in Romanticism Q-18 & & College X-165 \\
\hline SLang & Studies in Language 1-15 & Text & see $I-15$ \\
\hline SlavR & Slavic Review L-128 & TheatreS & Theatre Studies U-18 \\
\hline$s W J$ & School Library Journal L-179 & Thieme and Becker & see $\mathbf{A}-70$ \\
\hline SLJ & Southern Literary Journal S-18 & THJCS & $\begin{array}{l}\text { T sing-hua Journal of Chinese } \\
\text { Studies L-148 }\end{array}$ \\
\hline Slocum & $\begin{array}{l}\text { see G-1 } \\
\text { Speech }\end{array}$ & ThR & Theatre Research International U-18 \\
\hline $\begin{array}{l}S M \\
S M C\end{array}$ & $\begin{array}{l}\text { Speech Monographs I-15, X-165 } \\
\text { Studies in Medieval Culture N-18 }\end{array}$ & Thrall, Hibbard \& & \\
\hline SMed & Studi medievali N-18 & Holman & see $\mathrm{L}-12$ \\
\hline SN & Studia Neophilologica L-57 & ThS & Theatre Survey U-18 \\
\hline SNNTS & Studies in the Novel W-5, W-8 & $T H Y$ & Thomas Hardy Yearbook M-60 \\
\hline SOED & $\begin{array}{l}\text { Shorter Oxford English Dictionary on His- } \\
\text { torical Principles I-33 }\end{array}$ & $\begin{array}{l}\text { Tilley } \\
T L S\end{array}$ & $\begin{array}{l}\text { see } \mathrm{K}-56 \\
\text { Times Literary Supplement } \mathrm{Z}-151\end{array}$ \\
\hline SoR & Southern Review S-18 & $T N$ & Theatre Notebook U-18 \\
\hline SoSt & Southern Studies S-18 & Totok-Weitzel & see $A-23$ \\
\hline SovL & Soviet Literature L-128 & $T P A$ & $\begin{array}{l}\text { Thoung Pao: Revue internationale de } \\
\text { sinologie } \mathrm{L}-148\end{array}$ \\
\hline $\begin{array}{l}S P \\
S Q\end{array}$ & $\begin{array}{l}\text { Studies in Philology L-57 } \\
\text { Shakespeare Quarterly O-50, 0-58 }\end{array}$ & TPS & $\begin{array}{l}\text { Transactions of the Philological } \\
\text { Society } 1-15\end{array}$ \\
\hline$S \vec{R}$ & Sewanee Review Z-158 & $T Q$ & Theatre Quarterly U-18 \\
\hline SR & Stationers' Register C-6 & $T Q$ & Thoreau Quarterly S-50 \\
\hline SRen & Studies in the Renaissance $0-18$ & TRev & Translation Review I-106 \\
\hline SRO & $\begin{array}{c}\text { Shakespearean Research and } \\
\text { Opportunities O-58 }\end{array}$ & TRHS & $\begin{array}{l}\text { Transactions of the Royal Historical } \\
\text { Society F-10 }\end{array}$ \\
\hline SS & Scandinavian Studies L-108 & $T S$ & Today's Speech X-165 \\
\hline SSF & Studies in Short Fiction W-8, W-52 & $T S B$ & Thoreau Society Bulletin S-50 \\
\hline $\begin{array}{l}\text { SSI } \\
\text { SSL }\end{array}$ & $\begin{array}{l}\text { Short Story Index W-55 } \\
\text { Studies in Scottish Literature M-78 }\end{array}$ & $T S L L$ & $\begin{array}{l}\text { Texas Studies in Literature and } \\
\text { Language L-57 }\end{array}$ \\
\hline SSSL & $\begin{array}{l}\text { Society for the Study of Southern } \\
\text { Literature Z-25 }\end{array}$ & $T S W L$ & $\begin{array}{l}\text { Tulsa Studies in Women's } \\
\text { Literature L-189 }\end{array}$ \\
\hline StAH & Studies in American Humor S-18 & $T W I$ & Writing Instructor X-165 \\
\hline Stationers' Register & see $C-6$ & $T W T$ & Technical Writing Teacher X-165 \\
\hline STC & Short Title Catalogue C-5 & TxU & University of Texas Libraries B-35 \\
\hline$S t C$ & Studia Celtica M-93 & Type-Index & $\sec K-1$ \\
\hline STC & Short Title Catalogue C-5 & & \\
\hline STCL & $\begin{array}{l}\text { Studies in Twentieth Century } \\
\text { Literature R-18 }\end{array}$ & $\begin{array}{l}\text { UDC } \\
\text { Ulrichs }\end{array}$ & $\begin{array}{l}\text { Universal decimal classification A-21 } \\
\text { see D-100 }\end{array}$ \\
\hline STTH & $\begin{array}{l}\text { Science Technology \& the } \\
\text { Humanities Z-158 }\end{array}$ & $\begin{array}{l}\text { ULS } \\
\text { USC }\end{array}$ & $\begin{array}{l}\text { Union List of Serials D-91 } \\
\text { United States Catalogsee C-30 }\end{array}$ \\
\hline Style & $\sec I-15, X-165$ & UTO & University of Toronto Ouarterly L-57 \\
\hline SVEC & $\begin{array}{l}\text { Studies on Voltaire and the Eighteenth } \\
\text { Century P-18 }\end{array}$ & & Z-158 \\
\hline SWAL & Southwestern American Literature S-18 & Valázquez & see $I-75$ \\
\hline Symposium & see $\mathrm{L}-57$ & Venn & see $G-27$ \\
\hline & & Verbatim & see $1-15$ \\
\hline$T A$ & Theatre Annual U-18 & Vicaire & see $C-53$ \\
\hline Tanselle & see $\mathrm{C}-20$ & $V I J$ & Victorians Institute Journal Q-18 \\
\hline TAPA & $\begin{array}{l}\text { Transactions of the American Philological } \\
\text { Association } \mathrm{L}-48\end{array}$ & $\mathrm{ViU}$ & University of Virginia Libraries B-39 \\
\hline TArts & Theatre Arts Monthly U-18 & VLang & Visible Language I-15 \\
\hline TASJ & $\begin{array}{l}\text { Transactions of the Asiatic Society of } \\
\text { Japan L-148 }\end{array}$ & $\begin{array}{l}\text { VN } \\
\text { vol. }\end{array}$ & $\begin{array}{l}\text { Victorian Newsletter Q-18 } \\
\text { volume }\end{array}$ \\
\hline Taylor and Whiting & see $K-58$ & $V P$ & Victorian Poetry Q-18, Q-39 \\
\hline & & $V P R$ & Victorian Periodicals Review $Q-18, Q$ \\
\hline
\end{tabular}




\begin{tabular}{|c|c|}
\hline VQR & Virginia Quarterly Review Z-158 \\
\hline$V R$ & Vox Romanica $L-63$ \\
\hline VS & Victorian Studies Q-18 \\
\hline VWQ & Virginia Woolf Quarterly $\mathrm{M}-60$ \\
\hline W\&L & Women and Literature $\mathrm{L}-189$ \\
\hline WAL & Western American Literature S-18, S-117 \\
\hline Walford & see $A-21$ \\
\hline Watt & $\sec A-30$ \\
\hline WB & Weimarer Beitrdge L-108 \\
\hline $\boldsymbol{W C}$ & Wordsworth Circle M-60, Q-18 \\
\hline WCWN & William Carlos Willians Review S-50 \\
\hline Webster's 2nd & see $I-47$ \\
\hline Webster's 3rd & see $I-48$ \\
\hline Wellesley Index & $\sec \mathrm{D}-12$ \\
\hline Wells & see $N-30$ \\
\hline Wentworth & $\sec 1-54$ \\
\hline \multicolumn{2}{|c|}{ Wentworth \& Flexner see $1-55$} \\
\hline $\mathbf{W F}$ & Western Folklore $\mathrm{K}-23$ \\
\hline WHi & $\begin{array}{l}\text { Wisconsin Historical Society } \\
\text { Library H-27 }\end{array}$ \\
\hline WHIMSY & $\begin{array}{l}\text { World Humor and Irony Membership Serial } \\
\text { Yearbook W-157 }\end{array}$ \\
\hline Whitaker & see $C-15$ \\
\hline Whiting and Whiting & see $\mathrm{K}-55$ \\
\hline $\mathbf{W}$ & Writing Instructor $\quad X-165$ \\
\hline Wing & $\sec C-7$ \\
\hline Wing STC & $\sec C-7$ \\
\hline WJSC & $\begin{array}{l}\text { Western Journal of Speech } \\
\text { Communication X-165 }\end{array}$ \\
\hline $\mathbf{W L}$ & Women and Language $1-15$ \\
\hline WLSP & World List of Scientific Periodicals D-96 \\
\hline$W L T$ & World Literature Today L-148 \\
\hline WLWE & $\begin{array}{l}\text { World Literature Written in } \\
\text { English } \mathrm{M}-108\end{array}$ \\
\hline WPA & Writing Program Administration $\mathrm{X}-165$ \\
\hline WP & Words and Phrases Index I-30 \\
\hline Wright & $\sec I-41$ \\
\hline WS & $\begin{array}{l}\text { Women's Srudies: An Interdisciplinary } \\
\text { Journal L-189 }\end{array}$ \\
\hline WSJour & Wallace Stevens Journal S-50 \\
\hline WSL & $\begin{array}{l}\text { Die Welt der Slaven: Halbjahresschrift für } \\
\text { Slavistik L-128 }\end{array}$ \\
\hline WWR & Walt Whitman Review S-50 \\
\hline
\end{tabular}

\begin{tabular}{|c|c|}
\hline YCC & Yearbook of Comparative Criticism L-9 \\
\hline YCGL & $\begin{array}{l}\text { Yearbook of Comparative and General } \\
\text { Literature L-9 }\end{array}$ \\
\hline YER & Yeats-Eliot Review M-60 \\
\hline YES & Yearbook of English Studies M-25 \\
\hline YR & Yale Review Z-158 \\
\hline YWES & Year's Work in English Studies $\mathrm{M}-22$ \\
\hline YWMLS & $\begin{array}{l}\text { Year's Work in Modern Language } \\
\text { Studies L-52 }\end{array}$ \\
\hline YWOES & Year's Work in Old English Studies N-22 \\
\hline ZAA & $\begin{array}{l}\text { Zeitschriff für Anglistik und } \\
\text { Amerikanistik M-25 }\end{array}$ \\
\hline $\mathrm{ZCP}$ & Zeitschrift für celtische Philologie M-93 \\
\hline ZDA & Zeitschrifi fur deutsches Altertum $\quad \mathrm{L}-108$ \\
\hline ZDMG & $\begin{array}{l}\text { Zeitschrift der Deutschen } \\
\quad \text { morgenlandischen Gesellschafi L-148 }\end{array}$ \\
\hline $\mathbf{Z D P}$ & Zeitschrift für deutsche Philologie $\quad$ L-108 \\
\hline ZFSL & $\begin{array}{l}\text { Zeitschrift für französische Sprache und Li- } \\
\text { teratur L-78 }\end{array}$ \\
\hline $\mathbf{z f}$ & Zeitschrift für Volkstuande $\quad$ K-23 \\
\hline ZGL & $\begin{array}{l}\text { Zeitschriff für germanistische } \\
\text { Linguistik } \mathrm{L}-108\end{array}$ \\
\hline $\mathbf{Z R P}$ & $\begin{array}{l}\text { Zeitschrift für romanische } \\
\text { Philologie } \mathrm{L}-60, \mathrm{~L}-63\end{array}$ \\
\hline $\mathbf{Z S}$ & Zeitschrift für Slavistik L-128 \\
\hline ZSP & Zeitschrifi für slavische Philologie $\quad$ L-128 \\
\hline
\end{tabular}

For further help with abbreviations, acronyms, and sigla, see the following reference works:

Leistner, Otto. ITA: Internationale Titelabkürzungen von Zeitschriften, Zeitungen, wichtigen Handbüchern, Wörterbüchern, Gesetzen, u. s. w. 3rd ed. 2 vols. Osnabrick: Biblio Verlag, 1981.

Z6945.A2 LA

Wellington, Jean Susomey. Dictionary of Bibliographic Abbrevia. tions Found in the Scholarship of Classical Studies and Related Disciplines. Westport, Conn.: Greenwood, 1983.

PA99.W44

Alkire, Leland G. Periodical Title Abbreviations. 4th ed. 2 vols. Detroit: Gale, 1983.

Z6945.A2 W34 
